\title{
Study at 1,046 Megacycles per Second of the Reflection Coefficient of Irregular Terrain at Grazing Angles
}

\author{
Raymond E. McGavin and Leo J. Maloney
}

(February 12, 1959)

\begin{abstract}
An experimental determination of the reflection coefficient over rough terrain is reported. The reflected signal received over rough terrain is considered to be made up oft wo components, one that is a specular component and the other a Rayleigh-distributed component. Where one terminal is low, the Rayleigh component is considered to be small with respect to the specular component but increases in relative magnitude as the height of the lower terminal increases. A terminal height is reached where the specular component is no longer significant, and the reflected energy is essentially Rayleigh-distributed. A terminal height is quickly reached above which the mean value of the reflected energy is relatively constant, of a low value, and independent of the grazing angle.
\end{abstract}

\section{Introduction}

The interest in rough-terrain reflection has led to the publication of a large number of papers on this subject in recent years. The complexity of the problem is evidenced by the many different approaches to reasonable and usable solutions. The most obvious approach to the problem would be to consider rough-terrain reflection as a modification of smooth-terrain reflection (i.e., specular reflection) and attempt to express the rough-terrain reflection coefficient in terms of the smooth earth or Fresnel reflection coefficient. However, the problem is not at all that simple.

The field measured at a point in space above the earth and within the radio line-of-sight of the transmitter is the vector sum of a number of signal components arriving at the receiver over a number of different paths. In general, there is a single wave that arrives directly from the transmitter, while others arrive after being reflected at least once from the intervening ground. Ideally, where the ground is a smooth surface, modified image theory adequately describes the received field, which is the vector sum of a direct wave and a single groundreflected wave [1]. ${ }^{1}$ Where the ground contains known irregularities, few in number, and where the reflecting area is small, a reasonable estimate of the reflected energy can be determined on a Fresnel diffraction basis [2]. However, if the irregularities are numerous and haphazard and if the reflecting area is extensive, it is very difficult to determine Fresnel zones.

Some methods of treatment have been proposed [3 through 10] for a rough surface where an exhaustive knowledge of the surface is available, or where the surface is such that it can be approximated by a simple function or where the irregularities are small

1 Figures in brackets indicate the literature references at the end of this paper. with respect to the wavelength. In the absence of such idealized conditions, an analytical determination of the coefficient of reflection is not possible, and it has been common practice to assume specular reflection even over rough terrain when the reflection occurs near grazing relative to an average smooth ground.

Any successful theory would have to explain the inherent relationships between the pertinent parameters. The authors of this paper do not attempt to propound a theory to explain the physics of reflection from extensive randomly rough terrain having large irregularity, but do attempt to point out some relationships between these observed parameters. The approach must be statistical since it can be shown that, for such surfaces only this method leads to practical application [7]. Attention will be concentrated on grazing angles which are of greatest interest. The angle between the incident wave and the tangent to an approximating smooth surface at the point of reflection will be called the grazing angle if it is less than $5^{\circ}$.

\section{Experimental Procedure}

During August of 1954, a series of aircraft flights were made over transmission paths in eastern Colorado and western Kansas. Three paths were investigated: one originating at the Cheyenne Mountain transmitting site near Colorado Springs, Colo. passing through Haswell, Colo. and continuing beyond; another from. Pikes Peak through Haswell; and a third from Fort Carson through Haswell. The mean ground elevation of these paths varies from $6,200 \mathrm{ft}$ relative to mean sea level near Cheyenne Mountain to 4,200 ft in western Kansas. The object of the flights was to investigate the distribution of received field strengths along these paths at an operating frequency of $1,046 \mathrm{Mc}$ using horizontal polarization. A complete description of the transmission facilities can be found elsewhere [11]. Table 1 shows the pertinent data concerning the trans- 
mitting and receiving system. All flights with the exception of the $7,000-\mathrm{ft}$ and the $10,000-\mathrm{ft}$ flights on the Cheyenne Mountain path were flown in a westto-east direction. For the flights in the opposite direction, any difference in the antenna pattern and gain due to the required change in mounting was ignored, since absolute values of the field or even values of the field relative to each other for any two flights were not required. Diurnal effects in the characteristics of transmission have also been neglected since such effects would be expected to influence the values of reflection coefficient obtained only slightly. Likewise, standard air refraction is assumed; although the meteorological data collected at the time indicate some departure from a standard atmosphere, this also would not be expected to affect our conclusions appreciably.

Visual checkpoints were used to maintain the aircraft on the path and to locate the aircraft over the terrain. For purposes of calculation, it was assumed that the aircraft maintained a constant speed between checkpoints. This assumption seems warranted as the average speed for successive sectors did not vary by more than 5 percent.

For the most part, the entire region over which these measurements were made is very dry and is considered to be semiarid. A major portion of the ground is covered with Buffalo grass and sagebrush; a small part is under cultivation. The entire region is essentially without trees. Although there are isolated ranches and farms, there are no areas of concentrated population along the paths.

\section{Analysis}

\subsection{Interpretation of the Reflecting Surfaces of the Flight Paths}

Inasmuch as Rayleigh's criterion of roughness has been generally accepted as an estimate of the effective boundary between rough and smooth terrain, this criterion has been used in the following analysis.
Rayleigh's criterion of roughness,

$$
\Delta \phi=\frac{4 \pi}{\lambda} \Delta h \sin \psi,
$$

is an expression relating the root mean square deviations of the surface from a smooth surface $(\Delta h)$, the wavelength of the signal $(\lambda)$ and the grazing angle $(\psi)$. This phase difference $(\Delta \phi)$ of rays reflected from different levels of a surface determines the effective surface roughness. Critical values for this expression ranging from $\pi / 2$ to 0.1 have been used by various authors [12, 13, 14]. Beckman [7] uses this criterion to determine the existence of a horizontal reflecting element in a profile. He also uses a modification of the same expression to determine the classification of the surface reflection, from one that is characterized by the Dirac distribution as one extreme, to one that is characterized by the Rayleigh distribution as the other extreme. In any case, irrespective of the particular limiting value chosen, if only the area within the smooth-earth first Fresnel zone is investigated, all paths considered here must be classified as being rough at least for grazing angles greater than $0.4^{\circ}$, and for frequencies in excess of $1,000 \mathrm{Mc}$. It should be noted, however, that there is not exact correspondence between Beckman's consideration and that stated here. Beckman would consider a detailed analysis of the entire profile and the selection of horizontal elements. This method considers the average deviations from what is considered to be the principal reflecting area. A detailed surface analysis is not practical for areas as large as those involved in this study.

Another problem in the determination of the reflection coefficient is the consideration of divergence. When a wave is reflected from a curved smooth surface, the power density is decreased due to the spreading of the reflected wave. As the angle of the incident wave approaches grazing over a smooth curved surface, divergence causes the power density of the reflected wave to decrease to a very-low value. However, if the terrain is sufficiently rough, it is difficult to determine the effect of divergence, since

TABLE 1. Transmitting and receiving system data

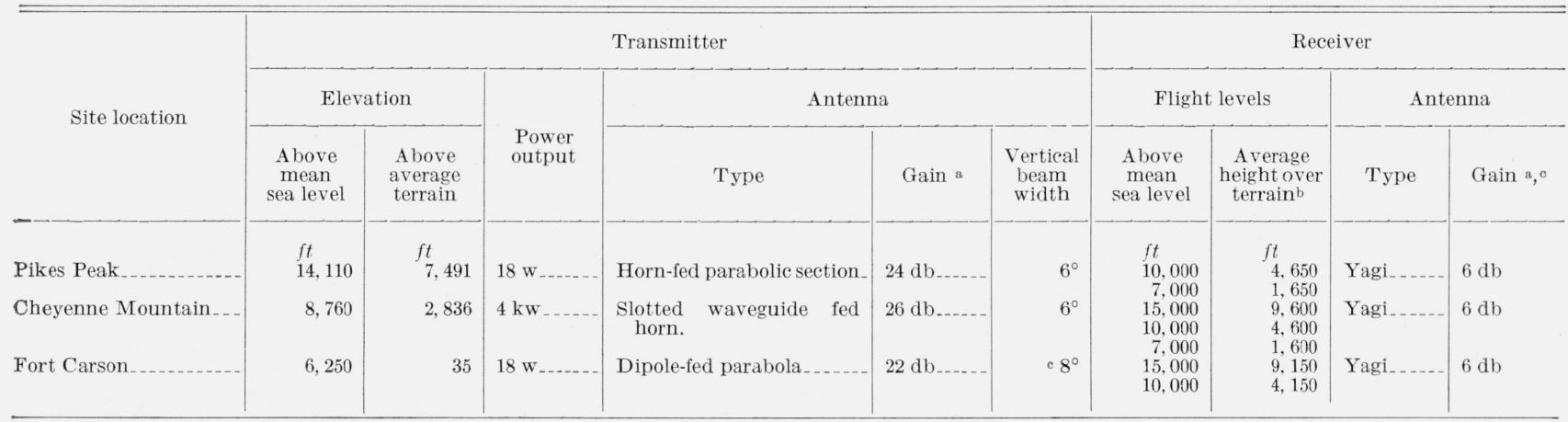

a Gains are relative to an isotropic radiator.

Calculated near the center of the path.

c Estimated.

$$
\begin{array}{lll}
\text { Note: } & \text { Operating Frequency: } & 1,046 \mathrm{Mc} \\
\text { Polarization: } & \text { Horizontal } \\
\text { Receiver: } & \text { A PR-5 aircraft } \\
\text { Aircraft: } & \text { B-17 }
\end{array}
$$


it would be reasonable to expect anomalies in the region of the radio horizon which would be more effective in determining the amount of reflection than would the general curvature of the surface at that point. Hence, the reflection coefficient spoken of here could be called the "effective reflection coefficient" as it has not been corrected for possible divergence caused by reflection from the curved surface of the earth.

In order to relate the results to a reflecting region on the ground, curves were fitted to the terrain and for the purposes of calculation of the grazing angles, the optical reflection point of the surface was assumed to lie on the curve rather than on the actual terrain [12]. In the case of the Pikes Peak and the Cheyenne Mountain paths, a single second order curve was drawn through the entire profile, and it was assumed that the curve was an approximation of the terrain, at least in a macroscopic sense. Since the terrain over the Fort Carson path did not permit a simple geometrical approximation, the results for the Fort Carson flights were related to the angle of elevation of the receiver above the transmitter radio horizon, rather than to the grazing angle.

\subsection{Characteristic Effects of Specular and Random Reflections}

When reflection from a smooth surface is considered, principles of optical reflection can be applied and the reflection can be viewed as though it occurred at a point. Then the reflection coefficient is the ratio of the electric field reflected to the electric field incident, considered along paths where the angle of reflection equals the angle of incidence. This reflection coefficient has both an amplitude and a phase, both of which are functions of the ground constants and the angle that the incidence ray makes with the tangent to the surface at the optical point of reflection. If it is assumed that the direct and ground-reflected waves arriving at the receiver left the transmitter with the same power density and traveled over paths approximately equal in length, the ratio of the amplitude of the reflected wave to the amplitude of the direct wave is equal to the amplitude of the reflection coefficient. Where the terrain is reasonably smooth, a well-defined "lobe structure" of field maximums and minimums is formed in space due to the interference between the direct and the ground-reflected waves. The accepted practice in evaluating the amplitude of a specular reflection coefficient over smooth terrain is to consider successive maximums and minimums of the field strengths of the received signal as a function of distance and to evaluate the reflection coefficient from the following expression:

$$
\rho=\frac{d_{2}}{d_{1}} \frac{\left(\frac{E_{M}}{E_{m}}\right)-1}{\left(\frac{E_{M}}{E_{m}}\right)+1},
$$

where:

$d_{1}=$ distance traveled by the direct wave,

$d_{2}=$ distance traveled by the reflected wave,

$E_{M}=$ the value of a voltage maximum, and

$E_{m}=$ the value of a preceding or following voltage minimum.

The well-defined maximums and minimums lend themselves well to the above method of analysis, as the reflected vrave can be considered to be a single ray. However, where the reflecting surface is very rough, such a model lobe structure does not exist. A moving receiver would experience fading at a more or less haphazard rate, and the validity of the above treatment is questionable. Since it is desirable to define the reflection coefficient over rough terrain in a manner similar to that over smooth terrain, the determination of the reflection coefficient should be in terms of grazing angles similar to those over the average smooth earth. If a least-square curve is substituted for the terrain and optical reflection points are assigned by geometry, the reflection coefficient can be defined as the electric field reflected to that incident along paths where the angle of incidence equals the angle of reflection over the average smooth earth. It should be remembered, however, that the reflected energy is not arriving via a single path anymore than it is in the case of specular or smooth-earth reflection as can be shown from a Fresnel diffraction treatment [2] of smooth plane-surface reflection. However, with rough terrain the problem is somewhat different since the reflected field becomes incoherent. Image theory is not adequate here, and the reflected energy, rather than being considered to consist of an essentially single reflected ray, must be considered more completely as consisting of the vector sum of many waves reflected from an extensive surface. The energy is diffused over a large region of space upon reflection and the reflected energy arriving at the receiver must be considered to be arriving from many different points on the reflecting surface at many different phase angles. The vector sum of these, if it is assumed that no one of the vectors is significantly large with respect to any of the others, would be a vector whose relative phase would be random and whose amplitude, $E_{s}$, would be distributed in accordance with the Rayleigh distribution [15]:

$$
\operatorname{Pr}\left(E_{s}>Z\right)=\exp \left(-Z^{2} / k^{2}\right)
$$

where $k^{2}=E_{1}^{2}+E_{2}^{2}+\ldots+E_{n}^{2}=\Sigma_{i=1}^{n} E_{1}^{2}=$ constant.

Then over rough terrain the signal arriving at the receiver can be interpreted as being the sum of a constant vector (the direct wave) and a Rayleigh distributed vector (the reflected wave). Perhaps this may seem to be a simplification of the problem in that it assumes that the surface reflection is characterized by a single distribution, namely the Rayleigh distribution. As was previously pointed out, at least one author [7] considers the Rayleigh distribu- 
tion to be the limiting case in so far as scattering in the principal direction (the direction in which specular reflection would take place if the surface were smooth) is concerned; i.e., it is required that the surface irregularities be quite large with respect to the wavelength. The two approaches are not wholly inconsistent, as it was pointed out that the departures of this terrain from a smooth surface are considerable even at small grazing angles.

\subsection{Separation of a Constant Vector and a Ray- leigh-Distributed Vector}

The sum of a direct wave and a wave reflected from a randomly rough surface will be considered to be composed of a constant component and a Rayleigh-distributed component, and can be separated into these two components by a consideration of the slope of the cumulative distribution of the received signal strength over discrete intervals of distance along the path $[15,16]$. The probability distribution of the amplitude $\left(r^{\prime}\right)$ of the sum of a constant vector plus a Rayleigh distributed vector was considered by Norton, Vogler, Mansfield, and Short [16], based on the expansion of a probability distribution derived by Rice [17] in the analysis of random noise:

$$
\operatorname{Pr}\left(r^{\prime}>r\right)=\frac{2}{k^{2}} \int_{r}^{\infty} r \exp \left[-\left(1+r^{2}\right) / k^{2}\right] I_{0}\left(2 r / k^{2}\right) d r
$$

where $r^{\prime}=$ instantaneous resultant amplitude, and where

$$
I_{0}(x)=\frac{1}{\pi} \int_{0}^{\pi} \exp (x \cos \phi) d \phi
$$

In figure 1, a relationship is given between the fading range in decibels $R(0.1)-R(0.9)$ and the level $K$ in decibels of the energy of the reflected wave relative to that of the direct wave. For example, when the ratio of the 10 percent to 90 percent values of the distribution of the received field over a discrete distance interval was $4.9 \mathrm{db}$, the average energy in the reflected wave would be expected to be $10 \mathrm{db}$ below that of the direct wave and this corresponds to an effective rms amplitude of the reflection coefficient of 0.316 . From such consideration, it can be seen that the reflection coefficient as defined above is expressed by a distribution and can have many different values. Any expression of a particular value must be made in terms of its statistical probability of occurrence. In this example 0.316 , the rms amplitude of the reflection coefficient, is that value which would be exceeded by 36.8 percent of all the possible values that the reflection coefficient may have; whereas the median value of the same reflection coefficient would be 0.263 which would be the value exceeded by 50 percent of all the values to be expected.

This theory, as can be seen from eq (2), assumes that the average energy of the reflected wave does not change appreciably from point to point within the distance intervals chosen. The distance intervals

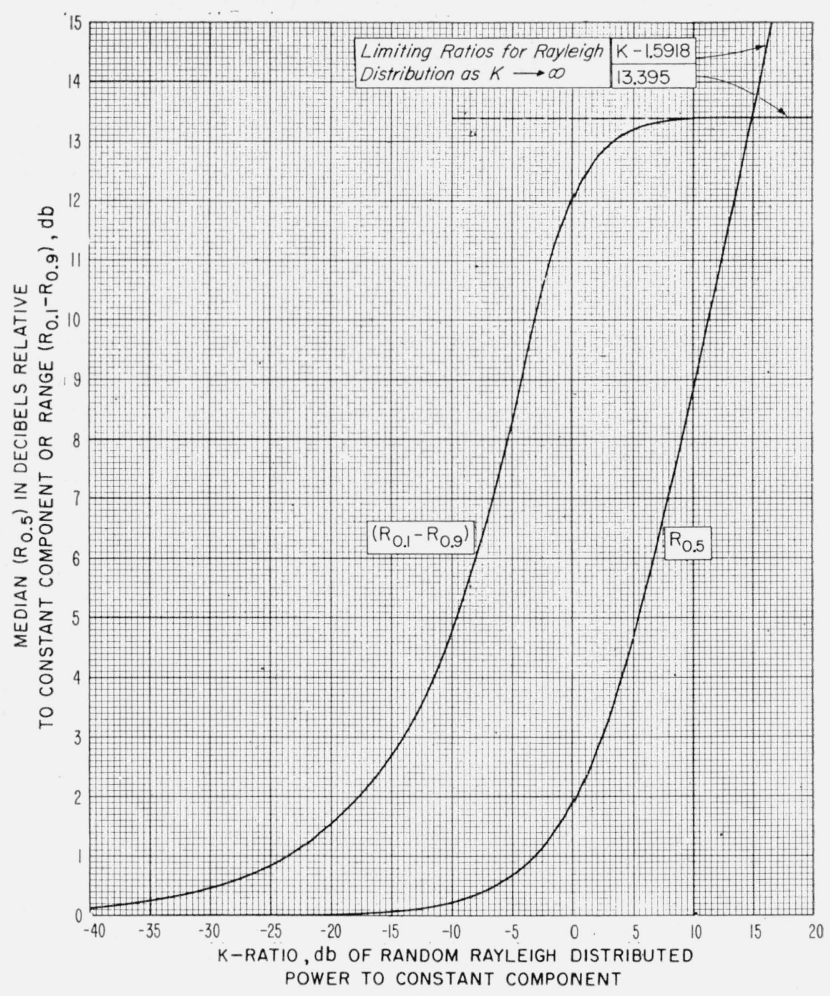

Figure 1. Median $\left(R_{0.5}\right)$ and range $\left(R_{0.1}-R_{0.9}\right)$ from the cumulative distribution of the resultant amplitude of a constant vector plus a Rayleigh-distributed vector.

Power in random component is $K \mathrm{db}$ relative to the range $\left(R_{0.1}-R_{0.9}\right)$ of the cumulative distribution.

were chosen somewhat arbitrarily to compromise the conflicting needs for sufficiently homogeneous data and sufficiently small sampling error. Intervals of one mile were chosen except close to the radio horizon where three, five and even ten mile intervals were used. For each interval, cumulative distributions of signal level versus distance were plotted on log-Rayleigh coordinates and the 10 percent and 90 percent values were found. The value $K$ was found from figure 1 for each value of $R(0.1)-R(0.9)$, and since $K=20 \mathrm{log} k$, each interval produced an estimate of the parameter $k$ of a Rayleigh distribution; that is, an estimate of the rms amplitude of the reflection coefficient. In the region of the radio horizon, as the grazing angle approaches zero, the spatial fading rate decreases and a quasi-lobe structure is formed. In this region it is easy to compare the two methods. For each interval, two values were determined; the rms value of several determinations of the reflection coefficient as determined considering successive maximums and minimums, and the rms value of a Rayleigh distribution for the interval assuming ground reflection to be random. The two different methods were then compared by use of the Wilcoxon signed rank test [18, p. 182]. Only two of the seven flights indicated statistically significant difference in the two methods. This comparison is mentioned not to question the validity of the approach presented here, but to point out its 
consistency with the conventional method in the interval near the horizon. It must be remembered that the conventional method assumes that the reflection coefficient does not change, at least in the interval between successive maximums and minimums. This requirement is more restrictive than the assumption that the average reflected energy does not change from period to period within the selected interval. Consequently, over rough terrain the random method seems to be a better approach.

\section{Results}

\subsection{Presentation of Results}

Figures 2, 3, and 4 are illustrations, both of the signal received in the aircraft, and the terrain profiles of the paths over which the flights were made. The dashed lines indicate the range of maximums and minimums which would be expected over the smooth earth determined by the curve fitted to the terrain. The calculated smooth earth lobe structure to be expected along these paths is also presented. It is easily seen that the Fort Carson path is not one that can be adequately approximated by a simple curve; hence, no comparable calculated values are presented for this path. From this presentation several characteristics of received fields over rough terrain are evident. When both terminals were very high, the regular lobe structure characteristic of specular reflection did not exist. Nor was the extensive range of maximums to minimums apparent. Contrasted to this, the Fort Carson path with a low transmitting terminal exhibited both a definite lobe structure and a more extensive range of maximums to minimums. It may be well to question whether the restricted fading range was actually present or might perhaps be due to the limitations of the frequency response of the recording system. An investigation of the data and of the frequency characteristics of the recording system indicated that the recorded maximums and minimums at the near end of the paths; i.e., at the points labeled 32 miles from the transmitter, would not differ from the actual maximums and minimums by more than 25 percent, this possible error being due to recorder response limitations. This possible error diminishes rapidly and from the points 42 miles from the transmitter to the radio horizon all the data appear to be well within the response limitations of the recording system.

This singularity of the Fort Carson path can be interpreted as the presence of an extensive surface which would support specular reflection. The profile of the Fort Carson path does show that two distinct surfaces between the transmitter and a point 5 miles from the transmitter are relatively flat, and the geometry indicates that reflected energy from these regions is received over all points of the flight path. However, the geometry of the entire profile indicated that multiple reflection from as many as four general regions of the surface contribute to the field experienced at a point. This is consistent with the appearance of the received signal; i.e., that the specular reflection produces lobing, while random reflection produces the higher frequency fluctuations superimposed. Consequently, it can be seen that if one terminal is low, small specular reflecting surfaces can be effective in producing the characteristic lobing of specular reflection; but if the terminal is raised sufficiently such that the specular reflecting surface becomes a small part of the illuminated zone, these surface anomalies become less effective and specular reflection less apparent. Thus, it is inferred that reflection from rough terrain contains two components, a specular component and a random component. Where one terminal is low the specular contribution can be significant, but this contribution will decrease as the height of the lower terminal is increased. Therefore, it is interpreted that the data from two paths are illustrations of the sum of only two significant components, a constant vector and a Rayleigh-distributed vector, while the Fort Carson data, in addition, include a significant groundreflected specular component.

Since two components in the ground reflection over the Fort Carson path can be identified, some method must be used to separate them. The difficulty of terrain approximation is such that no easy method can be devised based on path geometry. However, it seems reasonable to assume that the long-term spatial variations of the field are due only to specular reflection, and that the short-term spatial variations are the results of random reflection. If it can be assumed that the short-time rate-of-change of the effective amplitude due to the specular component is negligible in comparison to that due to the random component, then the medians of the instantaneous variations over short intervals accurately illustrate the relative field due to the specular component of the reflected waves plus the direct wave (fig. 5). Then the value of this specular component can be found using eq (1). It should be noted that this specular component is not necessarily a contribution from a single reflecting region, but is rather a summation resulting in a wave appearing as a single coherent wave at the receiver. Then, in this case, our "constant vector" is defined as consisting of a direct wave plus a coherent specular-reflected wave and to be slowly but uniformly changing due to the changing phase difference between the two coherent components. To compute the rms amplitude of the random reflection coefficient, a moving average can be used to eliminate the effect of the specular component and the analysis can then be done as outlined above.

Figures $6,7,8$, show the distributions of the estimates of the rms amplitude of the random reflection coefficient as a function of grazing angle for each flight as determined by the above method. It was pointed out previously that this treatment assumes that the average energy reflected is constant over the interval chosen; i.e., $k^{2}=$ constant.

The rms value of the random component was used only for convenience in that it was identically equal to $k$. The analysis would be approximately the same if mean values or median values were used, as 
TERRAIN PROFILE

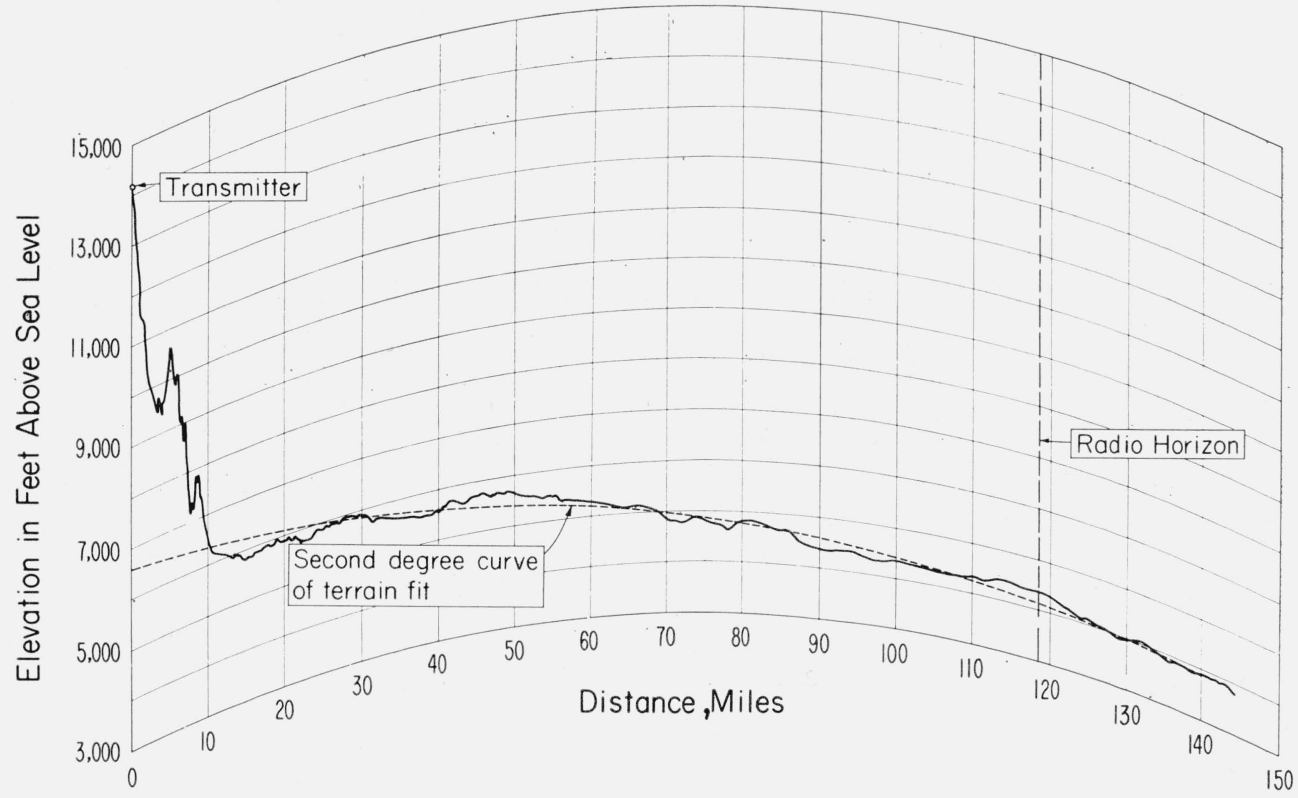

RECEIVED SIGNAL LEVELS

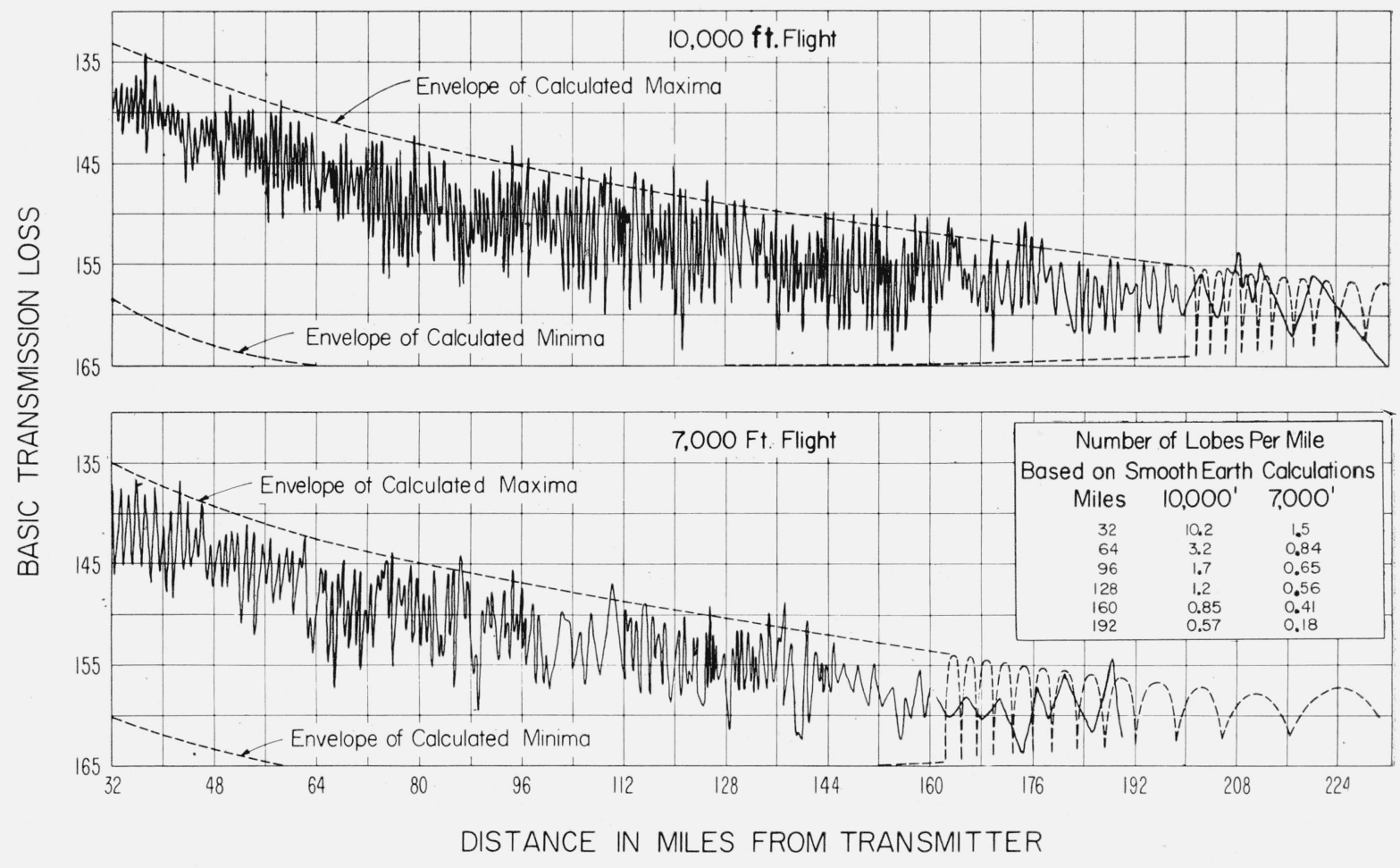

Figure 2. Pikes Peak to Haswell path. 


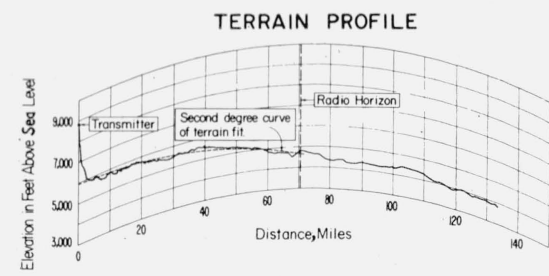

RECEIVED SIGNAL LEVELS
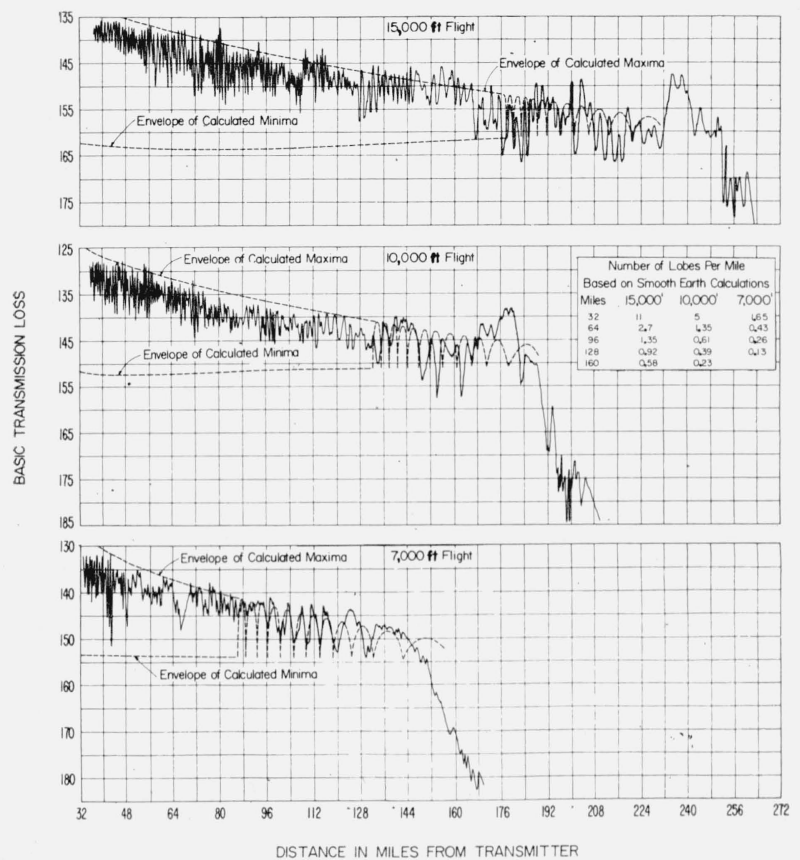

Figure 3. Cheyenne Mountain to Haswell path.

the Rayleigh distribution is completely specified when any one pertinent parameter is specified. For convenience here, the rms random component is to be interpreted as the rms value of the Rayleigh distribution. Since many considerations of Rayleigh distributions involve median values, it may be useful to note that the median value is equal to 0.8326 times the rms value.

\subsection{Interpretation of the Results as a Function of Grazing Angle and/or as a Function of Terminal Height}

It is reasonable to expect one or both of two possible relationships, the first, (a) that the rms random reflection coefficient is a function of the grazing angle, and/or the second, (b) that the rms random reflection coefficient is a function of the terminal height.

(a) To investigate the former, each flight was considered individually and regression analysis was performed to determine the trend. The regression coefficients of higher than the first degree terms were statistically insignificant; hence, linear regression was used. The linear regression lines are included on the appropriate figures 6 through 8 . Table $2, \mathrm{pt}$. (1), summarizes the statistical treatment of each individual flight as a function of the smooth earth grazing angle. Four of the seven correlation coefficients are statistically significant at the 5-percent probability level; however, two are positive and two are negative with no known explanation for the inconsistency. The significance may be unwarranted due to the fact that the observations may not be truly independent; whereas, the use of significance tests assumes independence. At any rate, the correlation coefficients are small, the largest being only 0.42 , indicating, that, in the extreme, only 18 percent of the variations in the reflection coefficient can be attributed to variations in the grazing angle. Therefore, it is reasonable to conclude that the rms random reflection coefficient was only slightly dependent on the grazing angles for grazing angles less than $5^{\circ}$.

(b) The second possibility is that the rms random reflection coefficient was a function of terminal height. Since in the previous consideration it was found that there was little relationship between the grazing angle and the value of these reflection coefficients, the logical approach here would be to test the hypothesis that there is no difference among the results of the different flights. This involves an analysis of variance and covariance. The process is somewhat tedious and only the results will be presented here. The reader is referred to any standard text on statistical analysis [18, ch. 7]. Four of the seven flights appear to come from a single population having a single mean and single variance. This appears to contradict the conclusion of significance for the correlation coefficients, since two of these flights (the flights on the Pikes Peak path) were found to have significant slopes. The Fort Carson data differ from the four flights in table 2, part (2), not due to the means but due to the larger variances. The Cheyenne Mountain, 7,000-ft flight results differ from the remainder, due both to the low value of the mean and to the excessive slope. The departure of the Cheyenne Mountain 7,000-ft data cannot be adequately explained. If, for the sake of convenience, this single flight is ignored the remainder of the data can be interpreted. The reasonable conclusion would be that with the lower terminal $35 \mathrm{ft}$ or higher, the mean value of the rms random component of the reflection coefficient does not vary with height in the range of heights investigated, but that increased variability about the mean was to be expected as this height approached $35 \mathrm{ft}$ as a lower limit. Hence, it appears that for the conditions of this experiment the only effect of change of the lower terminal height was to alter the degree of variability in the instantaneous value of the rms random component of the reflection coefficient about its mean value.

\subsection{Interpretation of the Results as Samples From a Single Population}

The absence of a substantial correlation between the above mentioned parameters suggests further consideration regarding the scatter of the observed results. 


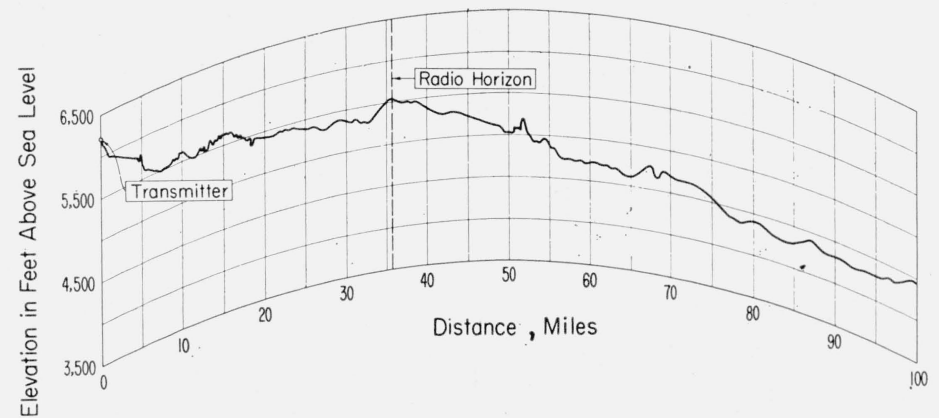

RECEIVED SIGNAL LEVELS

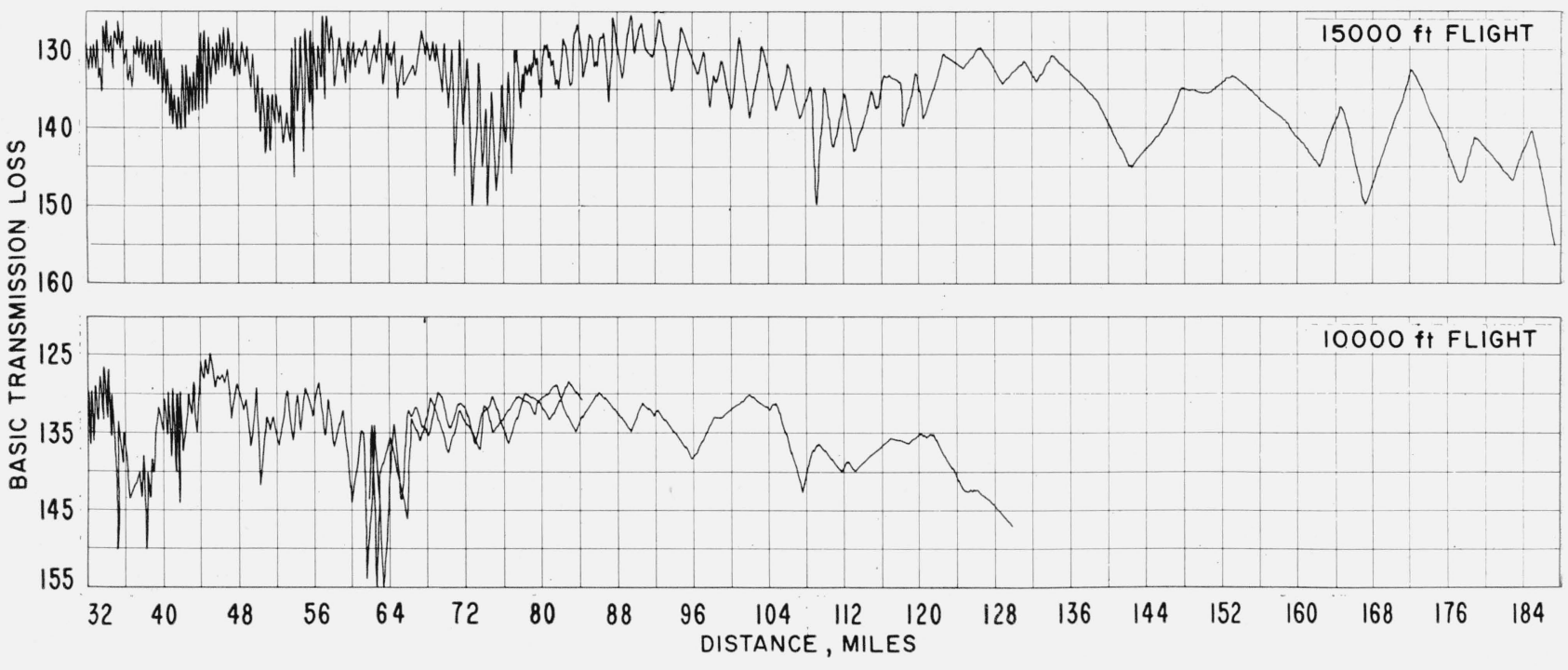

Figure 4. Fort Carson to Haswell path.

TABLE 2. Statistical parameters for the random component of the reflection coefficient

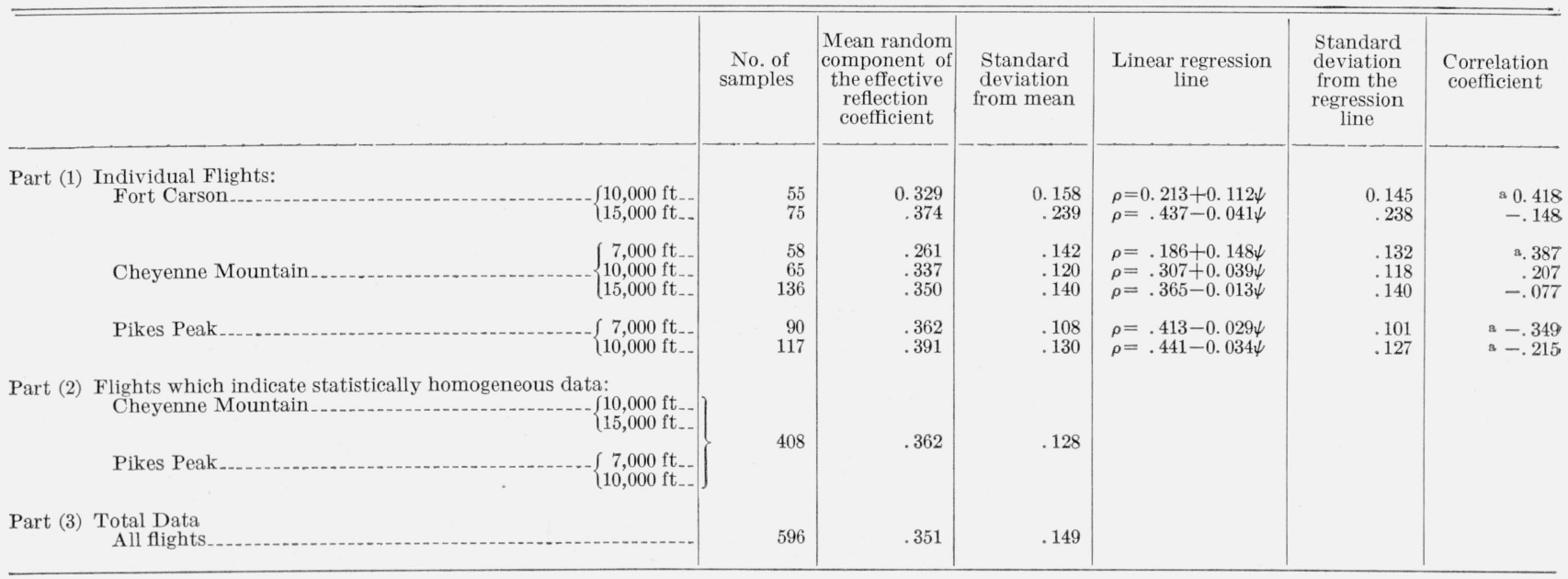

a Statistically significant at the $5 \%$ probability level. 

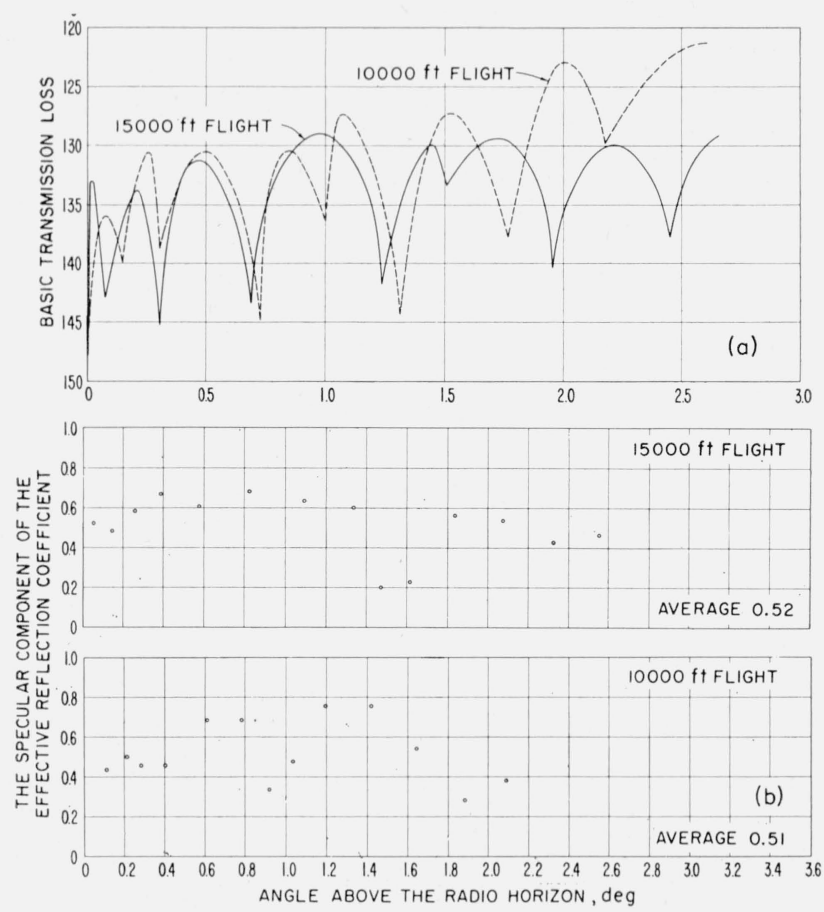

Figure 5. Fort Carson path.

a. Distribution of received signal showing only the long-term spatial variations attributed to a specular component. b. Distribution of the specular componen of the effective reflection coefficient

It should be remembered that each interval was analyzed as if it were an independent sample which is probably not strictly true, and that each "value" of the random reflection coefficient is an estimate of the rms value of a Rayleigh-distributed variate for that interval. Consequently, the results presented in figures 6,7 , and 8 are distributions of estimates of the rms values of a series of approximately independent Rayleigh distributions. It is reasonable

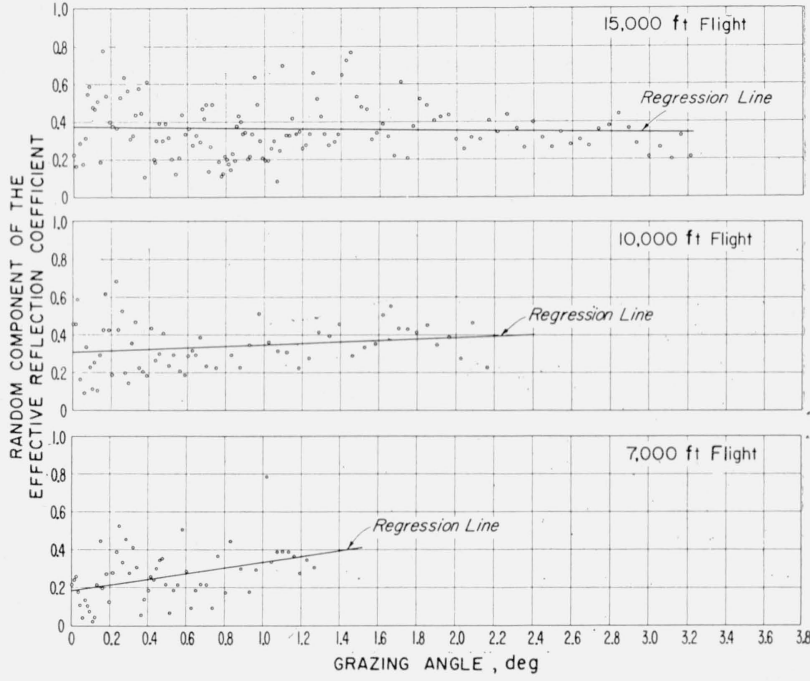

Figure 7. Cheyenne Mountain path.

Distribution of the effective reflection coefficient.

at this point to consider the possibility that these estimates of the rms value of all of these independent Rayleigh distributions may be normally distributed. This would be equivalent to saying that the form of the cumulative distribution would remain the same, but that the value of the intercept would be normally distributed. The method of analysis indicates a possibility that the estimates of any of three parameters may be approximately normally distributed. The parameter $K$, where $K=10 \log k^{2}$, could indicate a log-normal relationship; the reflected energy (proportional to $k^{2}$ ) may be normally distributed; or the rms random reflection coefficient (equal to $k$ ) may be normally distributed. Kolmogorov's test, as applied by Barrows and Norton [19], was used to test the normality of the distribu-

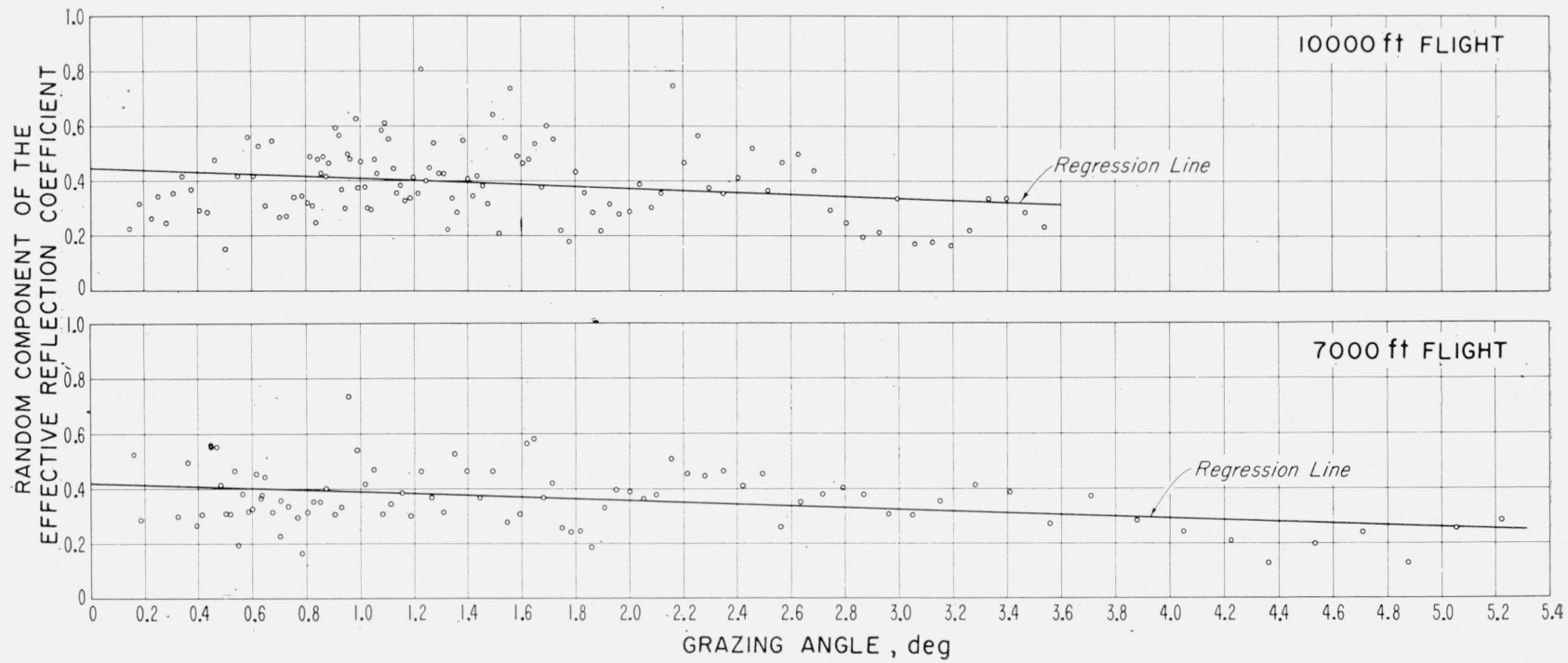

Figure 6. Pikes Peak path.

Distribution of the effective reflection coefficient. 


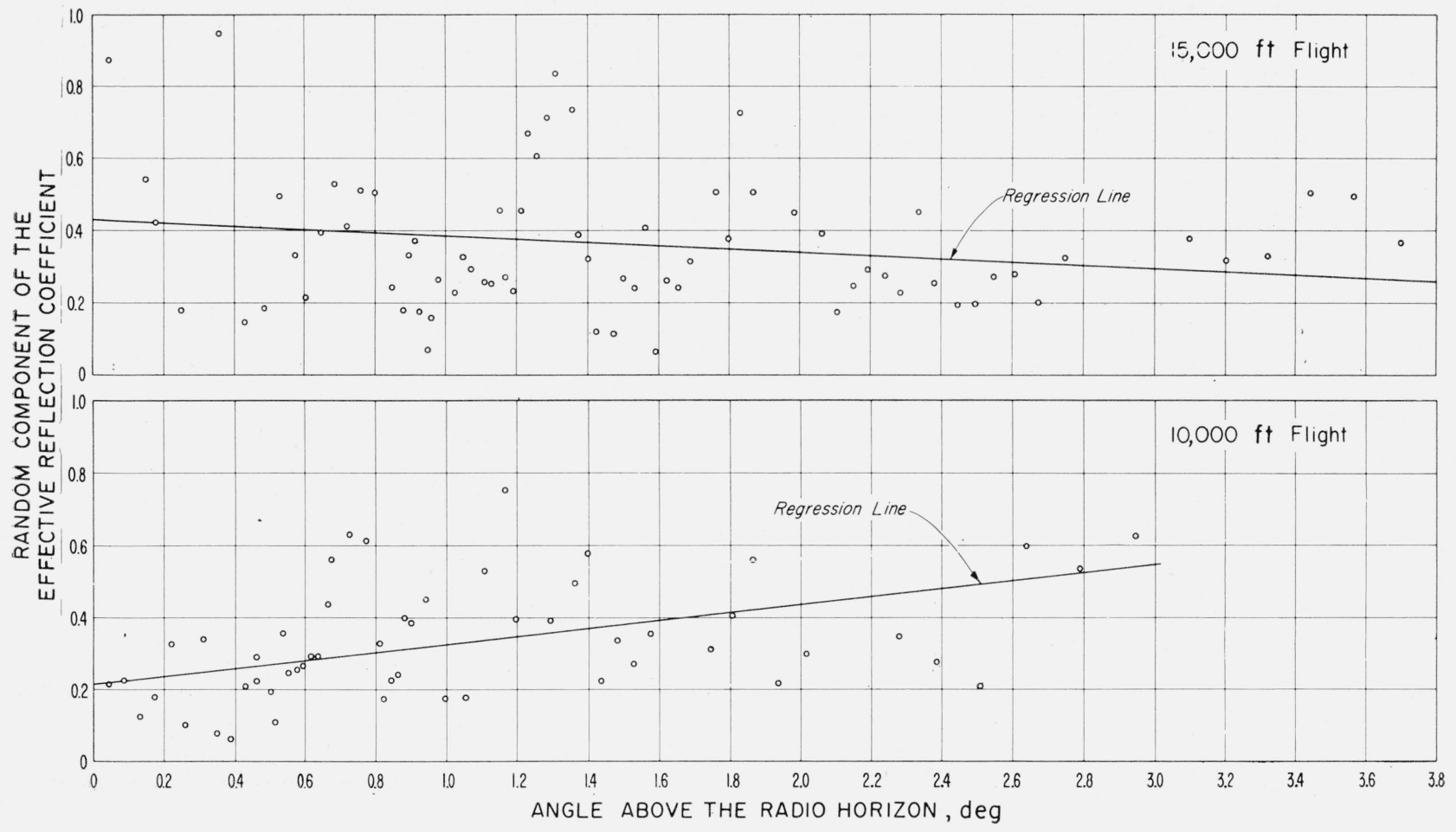

Figure 8. Fort Carson path.

Distribution of random component of the effective reflection coefficient based on short-term spatial variations of received signal.

tions of these three estimates. This is not an exact test, but a reliable approximation. The test was used in both directions; i.e., (1) on each flight as a separate unit, and (2) on each interval of grazing angle utilizing the data from all flights. As a result of testing each flight as a separate unit, and testing each interval of grazing angle, the normal distribution was found to approximate the distribution of $k$ better than the distributions of $k^{2}$ and $K$. When the data were considered as a whole, all three parameters appeared normally distributed, however, $k$ again proved to be the best approximation.

It was pointed out earlier that statistically these flights were not samples from the same population; consequently, when all of the data are considered as a unit, some error will be introduced. There are apparently three populations of data: the Cheyenne Mountain 7,000-ft data; the Fort Carson data; and the remainder which form a homogeneous group. The amount of error introduced by such an amassing should be relatively small. The lowcorrelation coefficients, even though significant, indicate that little error is introduced when the results of each flight are considered as samples of a distribution having a single mean. Considering the results of all flights as members of a single population requires only that it be remembered that the lower antennas introduce a higher variability from the mean value. (The departure of the Cheyenne Mountain 7,000-ft results is not explained.) Comparing the results of considering the data as a whole, with only that part of the data indicated to be homogeneous, little difference is noted. In the former case, the mean is 0.35 with a standard deviation of 0.15 for 596 observations; in the latter case, the mean is 0.36 with a standard deviation of 0.13 for 408 observations. Comparison of the amassed results can also be made up with the inhomogeneous group; i. e., the Fort Carson data where the 15,000-ft flight shows the greatest departure. The mean of this flight is 0.37 and the standard deviation is 0.24 , which illustrates the tendency for greater variability when one terminal is very low.

If this conclusion is utilized in two steps, further evidence of a lack of significance is seen. Considering first, that the random reflection coefficient is independent of terminal height, and considering that estimates of the rms reflection coefficient would be normally distributed within small intervals of the grazing angle, it is possible to reevaluate the random reflection coefficient as a function of the grazing angle. The mean and standard deviation was found for each interval of the grazing angle utilizing the estimates of $k$ from all seven flights. The result of this procedure are shown in figure 9 . The 90-percent confidence limits are only approximate since the data are not from a truly single population. The point at $1.3^{\circ}$ seems to be excessively high. This is due chiefly to the contributions from the Fort Carson data at this point, and can be interpreted as caused by possible anomalies in the Fort Carson path. It is in this region that the assumption of a single population is poor. It appears that the distribution of the Fort Carson 

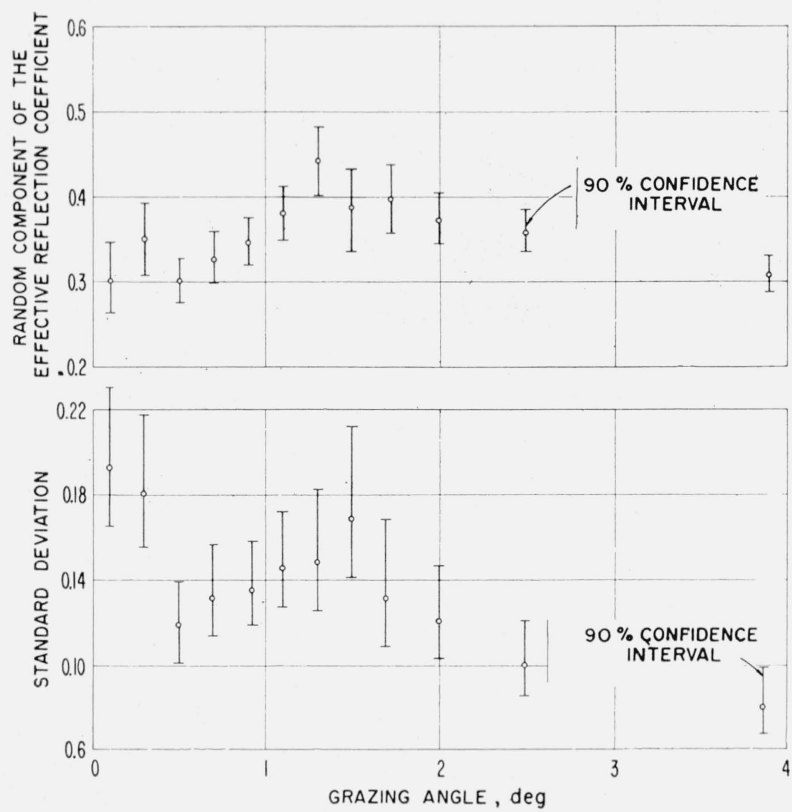

Figure 9. Random com ponent of the effective reflection coefficient as a function of the grazing angle for all fights.

data may be the sum of two normal distributions having different means; a departure such as this can be caused by the addition of a small number of nonidentical populations. Although this is indicated, it is not good statistical procedure to dissect data unless more justifiable reasons are evident [20].

As a second consideration, the reflection coefficient can be assumed to be independent of the grazing angle, and the estimates of the rms value of the random reflection coefficient can be considered to be normally distributed for discrete terminal heights. Here, the mean and standard deviation, as shown in table 2 for each flight, can be expressed as a function of transmitter height. Such results would reevaluate the random reflection coefficient as a function of terminal height. Figure 10 is an illustration of such a consideration. With the exception of the Cheyenne Mountain 7,000-ft flight indicated at a transmitter height of $2,836 \mathrm{ft}$ and a receiver height of $1,625 \mathrm{ft}$, there is little difference in the value of the random component of the reflection coefficient. It should be noted that the standard deviation decreased with increase of terminal height.

Kolmogorov's test applied to the total data confirmed the assumption of normality. Figure 11 illustrates the cumulative distribution of the total data. The mean for the total data is 0.35 and the standard deviation is 0.15 (from table 2), which compares well with the median value of 0.33 and a standard deviation of 0.15 (from fig. 11).

The proper interpretation of these results is that the rms random component here stated is the rms value of a Rayleigh distribution, and that the estimate of this rms value is approximately normally distributed having a mean value of 0.35 with a standard deviation of 0.15 . If the Rayleigh dis-
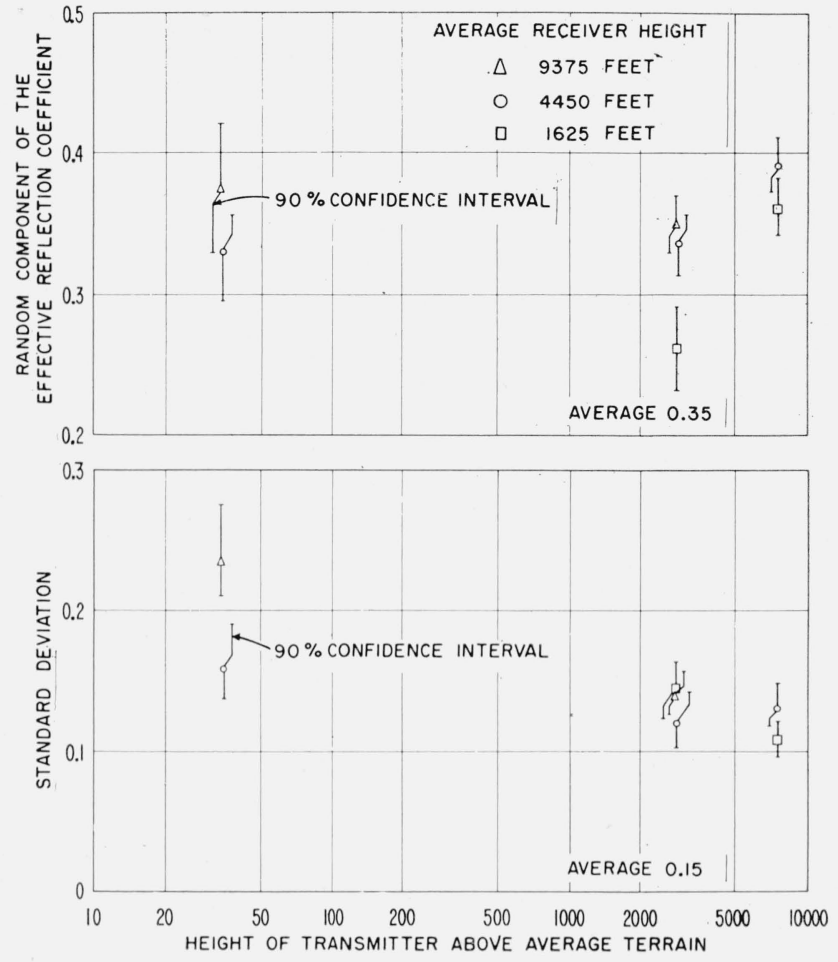

Figure 10. Random component of the effective reflection coefficient as a function of transmitter height for all flights.

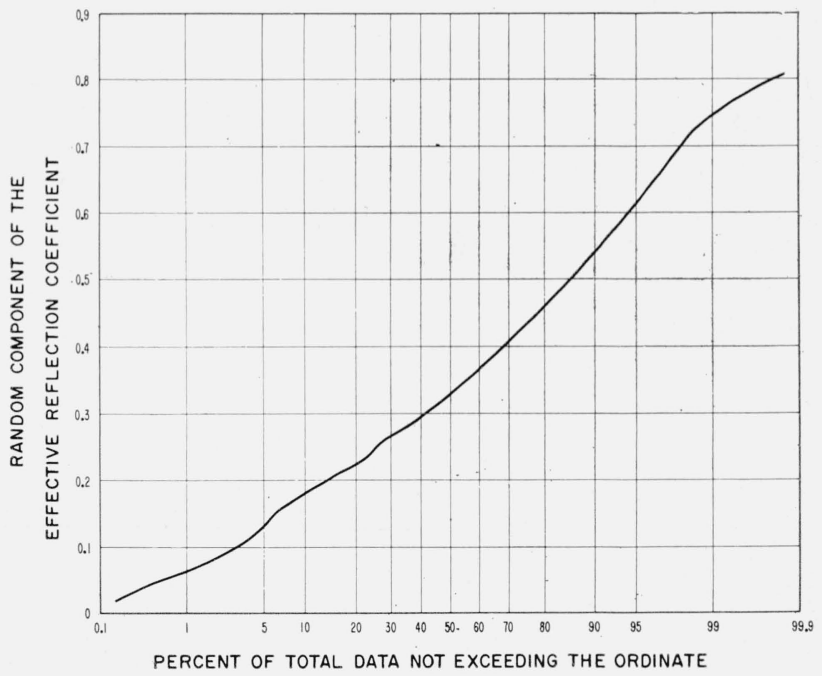

Figure 11. Cumulative distribution of the random component of the effective reflection coefficient.

Total data for all flights-596 points.

tribution were expressed graphically on log-Rayleigh coordinates, the slope would remain -1 , but the intercept at $\operatorname{Pr}(\rho>k)=0.368$ would be approximately normally distributed about 0.35 (fig. 12).

No doubt the standard deviation is a function of the length of the sampling interval as well as being a function of the different populations amassed 


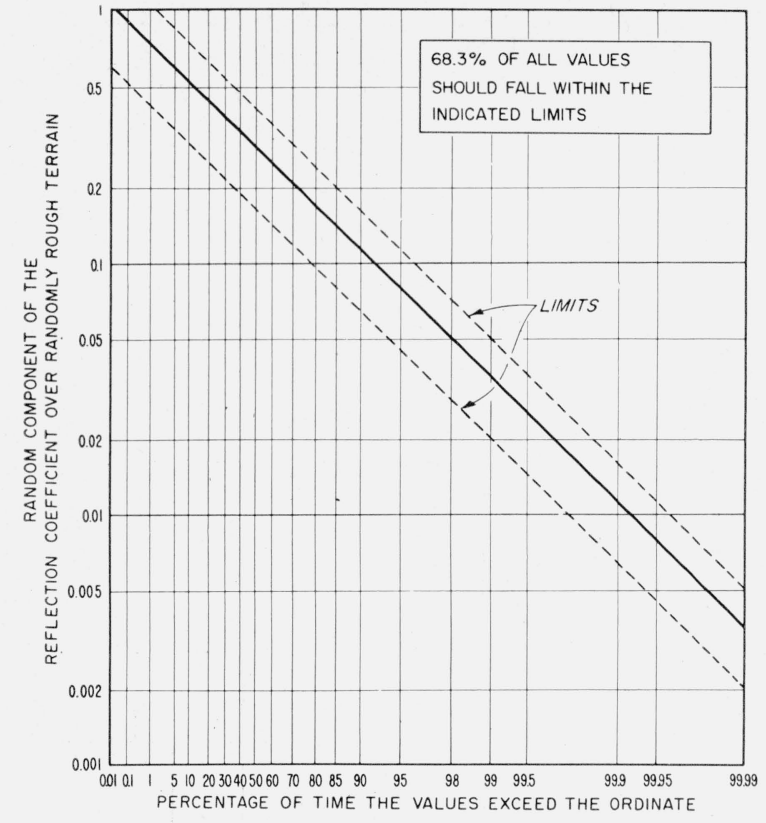

FIgURE 12. Distribution of random component of reflection coefficient for grazing angles less than $5^{\circ}$.

It is expected that 68.3 percent of individual values should be found to be between the indicated limits with the probability expressed along the abscissa.

in the total data. Thus it is difficult to interpret, i.e., it is composed of two parts; one is the variation of sample estimates of a population parameter, the rms random reflection coefficient; the other is the variation due to nonhomogeneous populations. No attempt is made here to indicate how much of this standard deviation is due to each source of variation.

It should be emphasized that this value for the random reflection coefficient ignores any divergency caused by reflection from the curved surface of the earth.

\section{Comparison With the Results of Other Investigations}

Due to the inability of accurately determining the reflecting surface, it was not possible to evaluate quantitatively the relationships expressed by some authors in terms of the type of analysis made here. However, in those cases where comparison was possible, remarkably good agreement was found particularly where overland measurements were considered.

Bullington's [21] data on the New York to Denver path compares favorably. Assuming a Rayleigh distribution for Bullington's data as suggested by Rice [5], we find a median value of 0.29 and an rms value of 0.35 . The variance is roughly the same. Straiton [22] utilizing four different frequencies over both land and water, achieved similar results for the higher frequencies over land. Similarity is found with the results of Sherwood and Ginzton [23] in the case of rough terrain. Although a proper comparison cannot be made with the results published by Beard, Katz, and Spetner [24], it is significant that comparable conclusions concerning the constancy of the random component with increasing effective roughness is also proposed by these authors. This constant mean value is quickly achieved for small values of the parameter expressing effective surface roughness. It should be noted that the results of these different investigators were found in widely separated places where ground constants can be assumed to differ. It would appear that the results are independent of the value of ground constants providing an overland path is considered.

In most cases where paths over rough water are considered, the value of the reflection coefficient for low grazing angles appears to be much higher and to approach more closely the smooth earth expected value $[7,22,23,24]$.

\section{Conclusion}

Thus, we arrive at the following description. The reflected signal received from rough terrain even at very small grazing angles can be considered to be made up of two components, one that is specular, and the other being Rayleigh distributed. Assuming one terminal as the reference, maintained above $1,000 \mathrm{ft}$, the relative magnitude of the two components of the reflection coefficient can be viewed as a function of the height of the variable terminal. Where the variable terminal is very low, the Rayleigh component may be considered to be small with respect to the specular component, but to increase in relative magnitude as the height of the low terminal is increased. Although in this experiment the rms random component exhibited essentially the same mean value for varying terminal heights, it would be expected that the value of the rms random component would approach zero as the height of the terminal approached zero [23]. The variance appears to decrease as the lower terminalheight increases especially at very low terminal heights. Therefore, the variability of the random component would be expected to be greater with a low terminal, diminishing as this terminal is raised. The overall mean value of the random component of the reflection coefficient was found to be 0.35 ; the standard deviation of individual estimates of the rms random component obtained from distance intervals of the flight paths as outlined above, was found to be 0.15 .

Although horizontal polarization was used in this investigation, S. O. Rice [5] points out that over rough terrain the identical mechanism is operative regardless of polarization. This view seems to be borne out in the overland data of Straiton [22] and Bianco and Morris [25], both of whom notice no polarization effect if the terrain is sufficiently rough. Thus, it is reasonable to assume that similar results would have been obtained along these paths if vertical polarization had also been investigated.

The results presented here viewed in comparison with the reports of others suggest further considera- 
tion of the problem of rough terrain reflection. It appears that there are many variables present which are not wholly understood. At present, it would appear that, over a spherical earth, if the terrain is sufficiently rough and sufficiently extensive, the value of the random reflection coefficient might be essentially independent of frequency, grazing angle, polarization, and ground constant; having a mean value of approximately 0.3 .

The variability of the instantaneous value is rather large and is more pronounced for low terminals. Specular reflection can be expected over rough terrain particularly if the terminals are low. There appears to be a difference between sea and land reflection in so far as rough terrain reflection is concerned, but at present it is not possible to determine whether this is due to marked difference in ground constants or to the degree of effective roughness.

It should be obvious that some liberty was taken with mathematical exactitude in the analysis of the data. This investigation points out what relationships appear to be present, but it should be apparent that additional study is required. Although it might appear that considerable data were present for this study, it is evident that a full investigation of this particular phenomenon will require considerably more than was available for this presentation. Such studies could lead to a possible macroscale factor for specifying rough terrain as a function both of frequency and antenna height.

\section{Addendum, An Estimate of the Relation- ship Between the Fading Range and Terminal Heights}

Since it is apparent that specular reflection was more effective than random reflection in providing large spatial fading ranges, it would be advantageous to determine a criterion for elimination of the specular component. Since it seems that low terminals produce a specular component even over rough terrain whereas high terminals produce only random reflection, an investigation of the relationship between terminal height and the prevalence of the specular component would be desirable. It is felt that the present data are not sufficiently complete to provide more than an indication. The spatial fading range for the Fort Carson data is interpreted to be $16.5 \mathrm{db}$ on the average. Since there has not been assumed geometrical relationship for the shape of the terrain, even the specular component is not corrected for divergence and the values expressed are effective values at these points. Regression analysis indicates that there was no significant correlation between the value of the effective specular reflection coefficient and the grazing angle. Hence, in computing the spatial fading range, the mean value of the specular component for the entire range (0.51) was used. The average maximum and minimum values of the sum of a direct wave and specularly-reflected wave whose coefficient is 0.51 were determined. Combination of these values with a random component having a proper rms value as expressed in table 2 vielded fading ranges of 16 and $17 \mathrm{db}$. (The spatial fading range as previously defined is the ratio of the fields exceeded $10 \%$ and $90 \%$ of short intervals of distance along the path.) Assuming that the higher terminals produce only random reflection with reflection coefficients as expressed in table 2, spatial fading ranges of less than $7 \mathrm{db}$ were encountered. Figure 13 is a preliminary estimate of the relationship between the spatial fading range and the height of the lower of two terminals (maintaining the other terminal above 1,000 ft) for grazing angles less than $5^{\circ}$. The unlabeled point is the average value of the spatial fading range for the $7,000-\mathrm{ft}$ flights. It would appear that the minimum spatial fading range to be expected under optimum conditions would be in excess of $5 \mathrm{db}$. If a fading range of $6 \mathrm{db}$ could be tolerated, then the minimum lower antenna height would be $600 \mathrm{ft}$, whereas $270 \mathrm{ft}$ would be sufficient for a 7 -db fading range. Of course, these relationships are based on the validity of the assumption of the shape of the curve of figure 13 . Many other curves including a straight line could be assumed with equal mathematical justification. The authors present this estimate as a reasonable approximation consistent with the data considered. Additional investigation is necessary before such an estimate could be accepted as typical of rough terrain phenomenon.

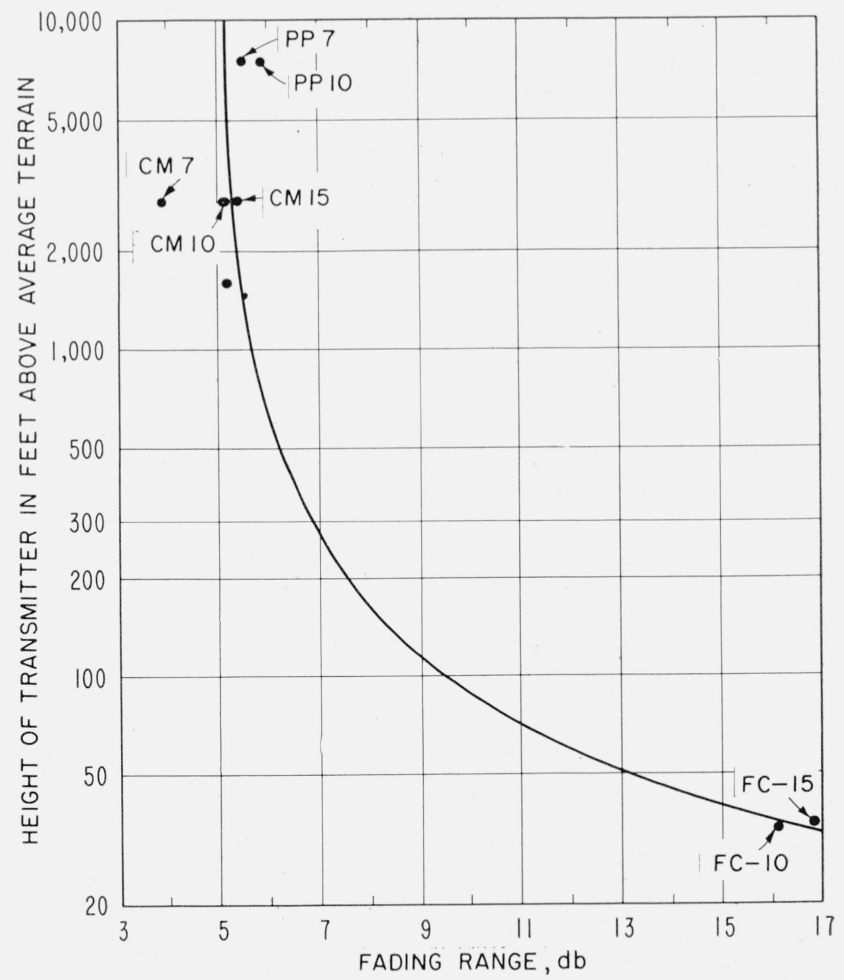

Figure 13. Estimate of the fading range as a function of terminal height over randomly rough terrain at 1,046 Mc for grazing angles less than $5^{\circ}$.

Receiver or reference terminal in excess of $1,000 \mathrm{ft}$. 
The authors acknowledge the cooperation of the Wright Development Center of the Wright-Patterson Air Force Base in supplying the B-17 aircraft. In addition, the authors acknowledge the assistance of Kenneth A. Norton, Jack W. Herbstreit, Kenneth O. Hornberg, and Dr. Edwin L. Crow of NBS, both in the presentation and in the review of this paper.

\section{References}

[1] R. S. Kirby, J. W. Herbstreit, and K. A. Norton, Service range for air-to-ground and air-to-air communications at frequencies above 50 megacycles, Proc. IRE 40, 535 (1952).

[2] K. A. Norton and A. C. Omberg, The maximum range of a radar set, Proc. IRE 35, 4 (1947).

[3] H. Davies, The reflection of electromagnetic waves from a rough surface, Proc. Inst. Elec. Engrs. (London) 101, Pt. IV, 209 (1954).

[4] V. Twersky, On the non-specular reflection of electromagnetic waves, J. Appl. Phy. 22, 825 (1951).

[5] S. O. Rice, Reflection of electromagnetic waves from slightly rough surfaces, Communs. Pure and Appl. Math. 4, 351 (1951).

[6] W. S. Ament, Toward a theory of reflection by a rough surface, Proc. IRE 41, 142 (1953).

[7] Peter Beckman, A new approach to the problem of reflection from a rough surface, Acta Tech. Ceskosl. Akad. Ved 2, 311 (1957).

[8] A. I. Kalinin, Approximate methods of calculating the field strength of ultra-short radio waves, taking into consideration the influence of the local terrain, Radio Tech. (Transl. Pergamon Inst.) 12, 13 (1957).

[9] M. A. Biot, Some new aspects of the reflection of electromagnetic waves on a rough surface, J. Appl. Phys. 28, 1455 (1957); Reflection on a rough surface from an acoustic point source, J. Acoust. Soc. Am. 29, 1193 (1957).

[10] J. R. Wait, Guiding of electromagnetic waves on a uniformly rough surface: parts I and II, Proc. International Symposium on Electromagnetic Theory, University of Toronto, June 15-20, 1959.

[11] A. P. Barsis, J. W. Herbstreit, and K. O. Hornberg, Cheyenne Mountain tropospheric propagation experiments, NBS Circ. 554, (1955).
[12] K. A. Norton, Transmission loss of space wave propagation over irregular terrain, IRE Trans. PGAP-3, 152 (1952).

[13] D. E. Kerr, Propagation of short radio waves, p. 396 (McGraw-Hill Book Co., New York, N.Y., 1951).

[14] C. R. Burrows and S. S. Attwood, Radio wave propagation, p. 466 (Academic Press, New York, N.Y., 1949).

[15] K. A. Norton, Propagation over rough terrain, U.S. NEL Symposium on Tropospheric Wave Propagation, July 25 to 29, 1949, Naval Electronics Rept. No. 173, p. 101.

[16] K. A. Norton, L. E. Vogler, W. V. Mansfield, and P. J. Short, The probability distribution of the amplitude of a constant vector plus a Rayleigh distributed vector, Proc. IRE 43, 1354 (1955).

[17] S. O. Rice, Mathematical analysis of random noise, Bell System Tech. J. 23, 282 (1944) and 24, 46 (1954).

[18] C. A. Bennett and N. L. Franklin, Statistical Analysis in Chemistry and the Chemical Industry (John Wiley \& Sons, New York, N.Y., 1954).

[19] E. Barrows and K. A. Norton, The Kolmogorov test of the goodness of fit of data samples to independently specified continuous distributions, together with an exact test of the normality of a small sample (to be published).

[20] A. Hald, Statistical theory with engineering applications, p. 152 (John Wiley \& Sons, New York, N.Y., 1952).

[21] K. Bullington, Reflection coefficients of irregular terrain, Proc. IRE 42, 1258 (1954).

[22] A. W. Straiton, Microwave radio reflection from ground and water surfaces, IRE Trans. PGAP-4, 37 (1952).

[23]. E. M. Sherwood and W. L. Ginzton, Reflection coefficients of irregular terrain at $10 \mathrm{~cm}$, Proc. IRE 43, 877 (1955).

[24] C.I. Beard, I. Katz, and L. M. Spetner, Phenomenological vector model of microwave reflection from the ocean, IRE Trans. AP-4, 162 (1956).

[25] D. R. Bianco and C. S. Morris, Radar terrain cross section at microwave frequencies, delivered at the fall Joint Meeting of URSI-IRE, Penn. State Univ., Univ. Park, Penn., (Oct. 20 to 22, 1958).

Boulder, Colo.

(Paper 63D2-20.) 